\title{
On Tubes of Force in a special class of electromagnetic fields.
}

\author{
By G. S. EAsTwOoD.
}

(Received 5th April 1923. Read 9th June 1923).

\section{Introduction.}

Professor Whittaker, in a paper entitled "On Tubes of Electromagnetic Force" (see Proceedings of the Royal Society of E'dinburgh, Vol. XLII., Part I. (No. 1)\}, introduces certain surfaces, which he names calamoids, in connection with an electromagnetic field in the four-dimensional world of space-time. The calamoids consist of "a convariant family of surfaces which when the field is purely electrostatic or purely magnetostatic reduce to the ordinary Faraday tubes of force." Professor Whittaker, in the paper referred to, also introduces two sets of surfaces, each a covariant family of $\infty^{2}$ surfaces, one of them named the electropotential surfaces, and the other family the magnetopotential surfaces of the electromagnetic field. The electropotential surfaces and the magnetopotential surfaces are shown to be everywhere absolutely orthogonal. (One member of each family meeting at a point, any line from this point in the one family is orthogonal to every line through the point in the other family). Moreover, a "calamoid, at every one of its points, is half-parallel and half-orthogonal to the electropotential surface which passes through the point, and is also half-parallel and half-orthogonal to the magnetopotential surface which passes through the point."

The electromagnetic field considered by Professor Whittaker is one in free space, and such that the electric and magnetic vectors are everywhere perpendicular to each other. In the last section (\$14), however, of the paper, he abandons the latter restriction and shows how to obtain two related families of geometrical forms in four-dimensional hyperspace involving the components of the electric and magnetic vectors of the field. Given certain conditions, "these geometrical forms are families of surfaces intersecting each other at right angles and resembling the electropotential and 
magnetopotential surfaces previously introduced." In this case "the calamoids can be introduced in precisely the same way" as before.

The aim in the following paper is to discuss the properties of the electromagnetic field after the manner indicated by Professor Whittaker in $\$ 14$, in the case when $d_{x}^{2}+d_{y}^{2}+d_{z}^{2}-h_{x}^{2}-h_{y}^{2}-h_{z}^{2}=0$ is satisfied everywhere and at all times, $d_{x}, d_{y}, d_{z}$ being the components of the electric vector and $h_{x}, h_{y}, h_{z}$ the components of the magnetic vector. When $d_{x} h_{x}+d_{y} h_{y}+d_{z} h_{z}=0$, i.e. when the electric and magnetic vectors are perpendicular, and when also $d_{x}^{2}+d_{y}^{2}+d_{z}^{2}-h_{x}^{2}-h_{y}^{2}-h_{z}^{2}$ is not zero, "the cross-section of a thin calamoid (measured by the area which it cuts off on the electropotential surfaces which intersect it in curves) multiplied by the value of $\left(d_{x}^{2}+d_{y}^{2}+d_{z}^{2}-h_{x}^{3}-h_{y}^{2}-h_{z}^{2}\right)^{\frac{1}{t}}$, is constant along the whole length of the calamoid." In what follows it will be shown that when $d_{x}^{2}+d_{y}^{2}+d_{z}^{2}-h_{x}^{2}-h_{y}^{2}-h_{z}^{2}$ is always zero and $d_{x} h_{x}+d_{y} h_{y}+d_{z} h_{z}$ is not zero, i.e. when the electric and magnetic vectors are not perpendicular, the quantity $\left(d_{x} h_{x}+d_{y} h_{y}+d_{z} h_{z}\right)^{\prime}$ multiplied by the cross section of a surface (defined as in $\$ 14$ of Professor Whittaker's paper) is constant throughout the length of a thin calamoid.

II. Eamilies of surfaces resembling the electropotential and magnetopotential surfaces.

Taking the set of total equations given by Professor Whittaker in $\$ 14$ of "On Tubes of Electromagnetic Force,"

$$
\left\{\begin{aligned}
\left(\lambda h_{z}+\mu d_{z}\right) d y-\left(\lambda h_{y}+\mu d_{y}\right) d z+\left(\lambda d_{x}-\mu h_{x}\right) d t & =0 \\
-\left(\lambda h_{z}+\mu d_{z}\right) d x+\left(\lambda h_{x}+\mu d_{x}\right) d z+\left(\lambda d_{y}-\mu h_{y}\right) d t & =0 \\
\left(\lambda h_{y}+\mu d_{y}\right) d x-\left(\lambda h_{x}+\mu d_{x}\right) d y+\left(\lambda d_{z}-\mu h_{z}\right) d t & =0 \\
-\left(\lambda d_{x}-\mu d_{x}\right) d x-\left(\lambda d_{y}-\mu h_{y}\right) d y-\left(\lambda d_{z}-\mu h_{z}\right) d_{z} & =0
\end{aligned}\right.
$$

and, also, the condition that this set shall be equivalent to only two equations, i.e.

$$
\begin{aligned}
& \left(d_{x} h_{x}+d_{y} h_{y}+d_{z} h_{z}\right) \lambda^{2}+\left(d_{x}^{2}+d_{y}^{2}+d_{z}^{2}-h_{x}^{2}-h_{y}^{2}-h_{z}^{2}\right) \lambda \mu \\
& -\left(d_{x} h_{x}+d_{y} h_{y}+d_{z} h_{z}\right) \mu^{2}=0,
\end{aligned}
$$

then, since $d_{x} h_{z}+d_{y} h_{y}+d_{z} h_{z}$ is not zero, while

$$
\begin{gathered}
d_{x}^{2}+d_{y}^{2}+d_{z}^{2}-h_{x}^{2}-h_{y}^{2}-h_{z}^{2}=0, \\
\mu= \pm \lambda ;
\end{gathered}
$$


hence, one family of geometrical forms is defined by the total differential equations

$$
\text { (A) }\left\{\begin{aligned}
\left(h_{z}+d_{z}\right) d y-\left(h_{y}+d_{y}\right) d z+\left(d_{z}-h_{z}\right) d t & =0 \\
-\left(h_{z}+d_{z}\right) d x+\left(h_{x}+d_{x}\right) d z+\left(d_{y}-h_{y}\right) d t & =0 \\
\left(h_{y}+d_{y}\right) d x-\left(h_{x}+d_{x}\right) d y+\left(d_{z}-h_{z}\right) d t & =0 \\
-\left(d_{x}-h_{x}\right) d x-\left(d_{y}-h_{y}\right) d y-\left(d_{z}-h_{z}\right) d z & =0
\end{aligned}\right.
$$

and another family of geometrical forms is defined by

$$
\text { (B) }\left\{\begin{aligned}
\left(h_{z}-d_{z}\right) d y-\left(h_{y}-d_{y}\right) d z+\left(d_{x}+h_{x}\right) d t & =0 \\
-\left(h_{z}-d_{z}\right) d x+\left(h_{x}-d_{x}\right) d z+\left(d_{y}+h_{y}\right) d t & =0 \\
\left(h_{y}-d_{y}\right) d x-\left(h_{x}-d_{x}\right) d y+\left(d_{z}+h_{z}\right) d t & =0 \\
-\left(d_{x}+h_{x}\right) d x-\left(d_{y}+h_{y}\right) d y-\left(d_{z}+h_{z}\right) d z & =0
\end{aligned}\right.
$$

Each of these two sets is a covariant set of differential equations. From set (A),

$$
\begin{aligned}
& d z=\frac{h_{x}-d_{x}}{d_{z}-h_{z}} d x+\frac{h_{y}-d_{y}}{d_{z}-h} d y \\
& d t=-\frac{h_{y}+d_{y}}{d_{z}-h_{z}} d x+\frac{h_{x}+d_{x}}{d_{z}-h_{z}} d y
\end{aligned}
$$

For these to be unconditionally integrable

$$
\left(\frac{\partial}{\partial x}+\frac{h_{x}-d_{x}}{d_{z}-h_{z}} \frac{\partial}{\partial z}-\frac{h_{y}+d_{y}}{d_{z}-h_{z}} \frac{\partial}{\partial t}\right) \frac{h_{y}-d_{y}}{d_{z}-h_{z}}-\left(\frac{\partial}{\partial y}+\frac{h_{y}-d_{y}}{d_{z}-h_{z}} \frac{\partial}{\partial z}+\frac{h_{x}+d_{z}}{d_{z}-h_{z}} \frac{\partial}{\partial t}\right) \frac{h_{x}-d_{z}}{d_{z}-h_{z}} \text { must }=0
$$
and also

$$
\left(\frac{\partial}{\partial x}+\frac{h_{x}-d_{z}}{d_{z}-h_{z}} \frac{\partial}{\partial z}-\frac{h_{y}+d_{y}}{d_{z}-h_{z}} \frac{\partial}{\partial t}\right) \frac{h_{z}+d_{x}}{d_{z}-h_{z}}-\left(\frac{\partial}{\partial y}+\frac{h_{y}-d_{y}}{d_{z}-h_{z}} \frac{\partial}{\partial z}+\frac{h_{z}+d_{x}}{d_{z}-h_{z}} \frac{\partial}{\partial t}\right) \frac{-\left(h_{y}+d_{y}\right)}{d_{z}-h_{z}} \text { must }=0
$$

Taking into account the Maxwellian equations,

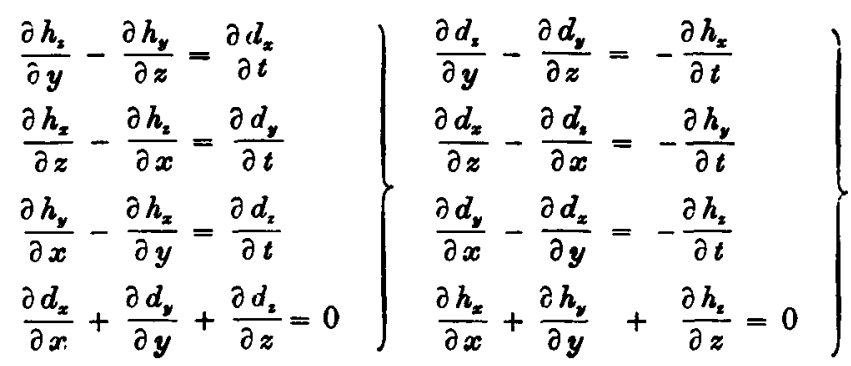


and also the fact that $d_{x}^{2}+d_{y}^{2}+d_{z}^{\mathrm{e}}-h_{x}^{2}-h_{y}^{2}-h_{z}^{2}$ is zero everywhere and at all times, it is found that these conditions of unconditional integrability are satisfied. Hence set (A) of total equations defines a family of $\infty^{2}$ surfaces. Similarly, for set (B), the conditions of unconditional integrability are satisfied and these equations also define a family of $\infty^{2}$ surfaces.

By the method of $\S 6$ of Professor Whittaker's paper the two families (A) and (B) can be shown to be absolutely orthogonal. For, taking the absolute of the four-dimensional hyperspace which is under consideration to be

$$
x_{1}^{2}+x_{2}^{2}+x_{3}^{2}-x_{4}^{2}=0, x_{5}=0,\left(x_{1}, x_{2}, x_{3}, x_{4}, x_{5}\right)
$$

being homogeneous coordinates and

$$
x=\frac{x_{1}}{x_{5}}, y=\frac{x_{2}}{x_{5}}, z=\frac{x_{3}}{x_{5}}, t=\frac{x_{1}}{x_{5}},
$$

the units being such that $c$, the velocity of light, is equal to 1 ; the condition that any two of the surfaces, one from each family, intersecting at a point, may be absolutely orthogonal, is

$$
d x_{1}, d x_{2}+d y_{1} d y_{2}+d z_{1} d z_{2}-d t_{1} d t_{2}=0,
$$

where $\left(d x_{1}, d y_{1}, d z_{1}, d t_{1}\right)$ is any line element from the point in the surface belonging to family (A) and $\left(d x_{2}, d y_{2}, d z_{2}, d t_{2}\right)$ is any line element issuing from the same point in the surface belonging to family (B).

Two lines are orthogonal when the points in which they meet the hyperplase at infinity are conjugate with respect to the absolute. This is satisfied by the two line elements if $d x_{1} d x_{2}+d y_{1} d y_{2}+d z_{1} d z_{2}-d t_{1} d t_{2}=0$. If the line elements are any that can be drawn in their respective surfaces from the given point of intersection, and if also this relation holde, then any line element from the point in one of the surfaces is perpendioular to overy line element from the point in the other surface; the surfaces are then abeolutely orthogonal.

$$
\begin{gathered}
\text { But, from set (A) }\left\{\begin{array}{l}
\left(h_{z}+d_{z}\right) d y_{1}=\left(h_{y}+d_{y}\right) d z_{1}-\left(d_{x}-h_{x}\right) d t_{1} \\
\left(h_{z}+d_{z}\right) d x_{1}=\left(h_{x}+d_{x}\right) d z_{1}+\left(d_{y}-h_{y}\right) d t_{1}
\end{array}\right. \\
\text { and from set (B) }\left\{\begin{array}{l}
\left(h_{z}+d_{z}\right) d z_{2}=-\left(h_{x}+d_{x}\right) d x_{2}-\left(h_{y}+d_{y}\right) d t_{2} \\
\left(h_{z}+d_{z}\right) d t_{2}=-\left(h_{y}-d_{y}\right) d x_{2}+\left(h_{x}+d_{x}\right) d y_{2}
\end{array}\right.
\end{gathered}
$$

If from these substitution is made in

$$
\left(h_{z}+d_{z}\right)\left(d x_{1} d x_{2}+d y_{1} d y_{2}+d z_{1} d z_{2}-d t_{1} d t_{2}\right),
$$


it is found to vanish. It will be found that if in this expression $\left(h_{z}+d_{z}\right)$ is replaced by $\left(d_{z}-h_{z}\right)$ or any other of the coeficients of the differentials in set (A) or set (B), the resulting expression vanishes and hence

$$
d x_{1} d x_{2}+d y_{1} d y_{2}+d z_{1} d z_{2}-d t_{1} d t_{2}=0 .
$$

This property of absolute orthogonality of families (A) and (B), as is mentioned in the introduction to the present paper, is stated by Professor Whittaker to be generally true ( $\$ 14$, last paragraph).

\section{The Calamoids.}

The partial differential equations of the calamoids can be set down at once since a calamoid is, at any one of its points, halfparallel and half-orthogonal to that member of family (A) which pusses through the point, and also half-parallel and half-orthogonal to that member of family (B) which passes through the point. Following $\$ 4$ and $\S 10$ of Professor Whittaker's paper, if we define any one of the surfaces of family (A) by the equations

$$
x=x(u, v), y=y(u, v), z=z\langle u, v), t=t(u, v)
$$

we get

$$
\frac{\frac{d_{z}-h_{z}}{\partial(x, y)}}{\frac{\partial(u, v)}{\partial(u, v)}}=-\frac{d_{y}-h_{y}}{\frac{\partial(x, z)}{\partial(u, v)}}=\frac{h_{x}+d_{x}}{\frac{\partial(x, t)}{\partial(u, v)}}=\frac{d_{x}-h_{x}}{\frac{\partial(y, z)}{\partial(u, v)}}=\frac{h_{y}+d_{y}}{\frac{\partial(y, t)}{\partial(u, v)}}=\frac{h_{z}+d_{z}}{\frac{\partial(z, t)}{\partial(u, v)}}
$$

and the direction ratios of this surface $(\alpha, \beta, \gamma, \delta, \epsilon, \zeta)$ are given by $\alpha: \beta: \gamma: \delta: \epsilon: \zeta$

$$
=\left(h_{z}+d_{z}\right):-\left(h_{y}+d_{y}\right):\left(d_{x}-h_{x}^{\prime}\right):\left(h_{x}+d_{x}\right):\left(d_{y}-h_{y}\right):\left(d_{z}-h_{z}\right) .
$$

Taking $(\alpha, \beta, \gamma, \delta, \epsilon, \zeta)$ for direction ratios of the tangent plane to a calamoid defined by

$$
x=x(u, v), y=y(u, v), z=z(u, v), t=t(u, v)
$$

we have $\alpha: \beta: \gamma: \delta: \epsilon: \zeta$

$$
=\frac{\partial(z, t)}{\partial(u, v)}:-\frac{\partial(y, t)}{\partial(u, v)}: \frac{\partial(y, z)}{\partial(u, v)}: \frac{\partial(x, t)}{\partial(u, v)}:-\frac{\partial(x, z)}{\partial(u, v)}: \frac{\partial(x, y)}{\partial(u, v)}
$$


Hence (see (10) and (15) of "On Tubes of Electromagnetic Force"), for the calamoid and the surface of family (A) to be halfparallel and half-orthogonal we have, respectively,

$$
\left\{\begin{array}{r}
\left(h_{z}+d_{z}\right) \frac{\partial(x, y)}{\partial(u, v)}-\left(h_{y}+d_{y}\right) \frac{\partial(x, z)}{\partial(u, v)}+\left(d_{x}-h_{x}\right) \frac{\partial(x, t)}{\partial(u, v)} \\
+\left(h_{x}+d_{x}\right) \frac{\partial(y, z)}{\partial(u, v)}+\left(d_{y}-h_{y}\right) \frac{\partial(y, t)}{\partial(u, v)}+\left(d_{z}-h_{z}\right) \frac{\partial(z, t)}{\partial(u, v)}=0 \\
-\left(d_{z}-h_{z}\right) \frac{\partial(x, y)}{\partial(u, v)}+\left(d_{y}-h_{y}\right) \frac{\partial(x, z)}{\partial(u, v)}+\left(h_{x}+d_{x}\right) \frac{\partial(x, t)}{\partial(u, v)} \\
-\left(d_{x}-h_{x}\right) \frac{\partial(y, z)}{\partial(u, v)}+\left(h_{y}+d_{y}\right) \frac{\partial(y, t)}{\partial(u, v)}+\left(h_{z}+d_{z}\right) \frac{\partial(z, t)}{\partial(u, v)}=0
\end{array}\right.
$$

These by addition and subtraction are at once seen to reduce to

$$
\begin{aligned}
& h_{z} \frac{\partial(x, y)}{\partial(u, v)}-h_{y} \frac{\partial(x, z)}{\partial(u, v)}+d_{x} \frac{\partial(x, t)}{\partial(u, v)} \\
&+h_{x} \frac{\partial(y, z)}{\partial(u, v)}+d_{y} \frac{\partial(y, t)}{\partial(u, v)}+d_{z} \frac{\partial(z, t)}{\partial(u, v)}=0 \\
&-d_{z} \frac{\partial(x, y)}{\partial(u, v)}+d_{y} \frac{\partial(x, z)}{\partial(u, v)}+h_{x} \frac{\partial(x, t)}{\partial(u, v)} \\
&-d_{x} \frac{\partial(y, z)}{\partial(u, v)}+h_{y} \frac{\partial(y, t)}{\partial(u, v)}+h_{2} \frac{\partial(z, t)}{\partial(u, v)}=0
\end{aligned}
$$

The partial differential equations satisfied by the calamoids are stated by Professor Whittaker ( $\$ 14)$ to be these in all cases.

(The results obtained in this section might have been obtained equally well by considering a calamoid as balf-parallel and halforthogonal to a member of family (B).)

\section{Integral Properties of the Calamoids.}

Apply the theorem that-in the four-dimensional hyperspace that is being considered, if $S$ be any closed surface to which we can fit an open hyperspace not containing electrons, then the integral

$$
\begin{aligned}
\iint\left\{h_{z} \frac{\partial(x, y)}{\partial(u, v)}-\right. & h_{y} \frac{\partial(x, z)}{\partial(u, v)}+d_{x} \frac{\partial(x, t)}{\partial(u, v)} \\
& \left.+h_{x} \frac{\partial(y, z)}{\partial(u, v)}+d_{y} \frac{\partial(y, t)}{\partial(u, v)}+d_{z} \frac{\partial(z, t)}{\partial(u, v)}\right\} d u d v
\end{aligned}
$$


vanishes when the integration is extended over the surface $S$ : and the integrai

$$
\begin{aligned}
\iint\left\{-d_{z} \frac{\partial(x, y)}{\partial(u, v)}\right. & +d_{y} \frac{\partial(x, z)}{\partial(u, v)}+h_{x} \frac{\partial(x, t)}{\partial(u, v)} \\
& \left.-d_{x} \frac{\partial(y, z)}{\partial(u, v)}+h_{y} \frac{\partial(y, t)}{\partial(u, v)}+h_{z} \frac{\partial(z, t)}{\partial(u, v)}\right\} d u d v
\end{aligned}
$$

vanishes when the integration is extended over the surface $S$-(see "On Tubes of Electromotive Force," p. 17) to the case of a thin calamoin closed at one end by a portion $s_{1}$ of one of the surfaces of set $(A)$ and at the other end by a portion, $s_{2}$, of another of these surfaces. Then, as each of the quantities within the brackets \{\} vanishes everywhere over the portion of $S$ formed by the calamoid, each integral vanishes when taken over both $8_{1}$ and $s_{2}$, the remaining part of $S$.

For the surfaces of which $s_{1}$ and $s_{2}$ are elements we have (as seen in Section III.),

$$
\frac{\frac{\partial(x, y)}{\partial(u, v)}}{d_{z}-h_{z}}=-\frac{\frac{\partial(x, z)}{\partial(u, v)}}{d_{y}-h_{y}}=\frac{\frac{\partial(x, t)}{\partial(u, v)}}{h_{x}+d_{x}}=\frac{\frac{\partial(y, z)}{\partial(u, v)}}{d_{x}-h_{x}}=\frac{\frac{\partial(y, t)}{\partial(u, v)}}{h_{y}+d_{y}}=\frac{\frac{\partial(z, t)}{\partial(u, v)}}{h_{z}+d_{z}},
$$

and each ratio

$$
=\frac{\left\{\left[\frac{\partial(x, y)}{\partial(u, v)}\right]^{2}+\left[\frac{\partial(x, z)}{\partial(u, v)}\right]^{2}+\left[\frac{\partial(y, z)}{\partial(u, v)}\right]^{2}-\left[\frac{\partial(x, t)}{\partial(u, v)}\right]^{2}-\left[\frac{\partial(y, t)}{\partial(u, v)}\right]^{2}-\left[\frac{\partial(z, t)}{\partial(u, v)}\right]^{2}\right\}^{t}}{\left\{\left(d_{x}-h_{x}\right)^{2}+\left(d_{y}-h_{y}\right)^{2}+\left(d_{z}-h_{z}\right)^{2}-\left(h_{x}+d_{x}\right)^{2}-\left(h_{y}+d_{y}\right)^{2}-\left(h_{z}+d_{z}\right)\right\}^{2}} .
$$

Hence, as the numerator of the last fraction multiplied by $d u d v$ gives the area of an element of surface defined by

$$
x=x(u, v), y=y(u, v), z=z(u, v), t=t(u, v),
$$

then considering element $s_{1}$

$$
\frac{\partial(x, y)}{\partial(u, v)} d u d v=\frac{d_{z}-h_{z}}{\left\{-4\left(d_{x} h_{x}+d_{y} h_{y}+d_{z} h_{z}\right)\right\}^{\frac{1}{3}}} \times \text { area of } s_{1},
$$

with five other corresponding relations; in like manner, similar relations obtain for $s_{2}$, the values of the vectors and of the 
coordinates being those belonging to the respective areas. Combining these with either of the integral relations given at the beginning of this section we bave

$$
\begin{aligned}
& \text { value of }\left(d_{x} h_{x}+d_{y} h_{y}+d_{z} h_{z}\right)^{\frac{1}{2}} \text { at } s_{1} \times \text { area of } s_{1} \\
= & \text { value of }\left(d_{x} h_{x}+d_{y} h_{y}+d_{x} h_{z}\right)^{\frac{1}{t}} \text { at } s_{2} \times \text { area of } s_{2}
\end{aligned}
$$

Thus, we have finally $\left(d_{x} h_{x}+d_{y} h_{y}+d_{z} h_{z}\right)^{\frac{1}{2}}$ multiplied by cross section of calamoid \{which consists of area cut off by any of the surfaces belonging to family (A)\} is constant along the calamoid. We might, of course, bave taken the areas of cross section as formed by two of the members of family (B) with the same result. A certain duality may thus be noted in connection with the properties of the calamoids depending on the vanishing of one or other of the quantities

$$
d_{x}^{2}+d_{y}^{2}+d_{z}^{2}-h_{x}^{2}-h_{y}^{2}-h_{z}^{2} \text { and } d_{x} h_{x}+d_{y} h_{y}+d_{z} h_{z} .
$$

